\title{
Quantum Dynamics with Spatiotemporal Control of Interactions in a Stable Bose-Einstein Condensate
}

\author{
Logan W. Clark, "Li-Chung Ha, Chen-Yu Xu, and Cheng Chin \\ James Franck Institute, Enrico Fermi Institute and Department of Physics, University of Chicago, Chicago, Illinois 60637, USA
}

(Received 17 June 2015; published 5 October 2015)

\begin{abstract}
Optical control of atomic interactions in quantum gases is a long-sought goal of cold atom research. Previous experiments have been hindered by rapid decay of the quantum gas and parasitic deformation of the trap potential. We develop and implement a generic scheme for optical control of Feshbach resonances which yields long quantum gas lifetimes and a negligible parasitic dipole force. We show that fast and local control of interactions leads to intriguing quantum dynamics in new regimes, highlighted by the formation of van der Waals molecules and localized collapse of a Bose condensate.
\end{abstract}

DOI: 10.1103/PhysRevLett.115.155301

PACS numbers: 67.85.-d, 03.75.Kk, 34.50.-s

Spatiotemporal control of interactions should bring a plethora of new quantum-mechanical phenomena into the realm of ultracold atom research. Temporal modulation of interactions is theoretically proposed as a route for creating anyonic statistics in optical lattices $[1,2]$ as well as new types of quantum liquids [3,4] and excitations [5-7]. Spatial modulation should grant access to unusual soliton behavior [8,9], controlled interfaces between quantum phases [10], stable nonlinear Bloch oscillations [11], and even the dynamics of acoustic black holes [12]. The conventional technique for controlling interactions in cold atoms, magnetic Feshbach resonance [13,14], is typically insufficient for these applications because magnetic coils are generally too large for very fast or local modulation.

A promising alternative is optical control of Feshbach resonances (OFR), with which high speed, spatially resolved control of interactions can be realized. Efforts toward achieving OFR in quantum gases have made significant progress [15-28] but have encountered two major obstacles. First, in previous experiments OFR has limited the quantum gas lifetime to the millisecond time scale $[24,26,27]$ due to optical excitation to molecular states. Short lifetimes forbid studies of quantum gases in equilibrium or after typical dynamical time scales. Second, the change of interaction strength from OFR is often accompanied by an optical potential. This potential can result in a parasitic dipole force which dominates the dynamics when the interactions are spatially modulated [21].

In this Letter we propose and implement a scheme for optically controlling interactions while maintaining a long quantum gas lifetime and negligible parasitic dipole force. With a far-detuned laser, a change of the scattering length $a$, which determines the interaction strength, by $180 \mathrm{Bohr}$ radii $\left(a_{0}\right)$ is only coupled with a slow radiative loss of $1.6 \mathrm{~s}^{-1}$. This loss rate is sufficiently low to allow the BEC to remain in equilibrium. Furthermore, the laser operates at a magic wavelength to eliminate the atomic dipole potential. We apply OFR to test the response of Bose-Einstein condensates (BECs) to rapid oscillation of interactions down to the time scale of $10 \mathrm{~ns}$, reaching beyond the van der Waals energy scale. Moreover, by spatially modulating the interaction strength we observe the intriguing dynamics brought on by localized collapse of the condensate, including the transient formation of solitons.

We optically control Feshbach resonances by using a fardetuned laser to light shift molecular states near the atomic scattering threshold (Fig. 1). The large detuning from all atomic and molecular transitions offers low heating and loss rates for the quantum gas. For a laser with intensity $I$, the total light shifts of atoms (subscript $a$ ) and molecules (subscript $m$ ) are given by [29]

$$
\begin{aligned}
\delta E_{a} & =\left(\alpha_{a}+\beta_{a} \mu_{a}\right) I, \\
\delta E_{m} & =\left(\alpha_{m}+\beta_{m} \mu_{m}\right) I,
\end{aligned}
$$

where $\alpha$ is the scalar polarizability and the vector polarizability $\beta \mu$ depends on the magnetic moment $\mu$. The

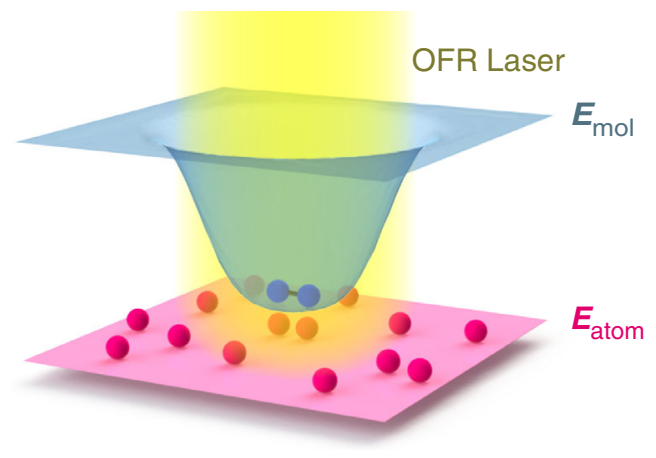

FIG. 1 (color online). Illustration of optical control of Feshbach resonances. A Feshbach resonance occurs when a laser (yellow) brings a molecular energy level (blue surface) close to the atomic scattering threshold (red surface). Here, the atom-molecule coupling makes atomic interactions more attractive at higher laser intensity. When operating at the magic wavelength, the beam does not shift the energy of single atoms (see text). 
polarizabilities can be calculated based on the laser detuning and the polarization-dependent dipole matrix elements of the transitions to the excited states [30]. Since our target molecular states are very weakly bound, they have similar polarizability to free atoms: $\alpha_{m} \approx 2 \alpha_{a}$ and $\beta_{m} \approx \beta_{a} \equiv \beta$. Assuming the molecular and atomic magnetic moments differ $\mu_{m} \neq 2 \mu_{a}$, the vector light shift can bring the molecular states closer to the scattering state, inducing a resonant atom-molecule coupling. Optical shifts of a magnetic Feshbach resonance have been observed using specific bound-to-bound transitions [25-28] and recently using a far-detuned laser [38]. Since our scheme does not rely on proximity to any atomic or molecular transitions, the lifetime is only limited by the one-body off-resonant scattering rate. Moreover, we choose a magic wavelength $\lambda_{M}$ to eliminate the dipole force on the atoms $\left(\delta E_{a}=0\right)$ [39], such that only the molecular shift

$$
\delta E_{m} \approx \beta\left(\mu_{m}-2 \mu_{a}\right) I
$$

remains (Fig. 1). Under these conditions the laser can change the scattering length without creating parasitic dipole forces. This scheme can be implemented in atomic species with a magnetic Feshbach resonance and a magic wavelength far-detuned from electronic transitions [30].

We implement this scheme for OFR using BECs of cesium atoms. Our experiment starts with precooled atoms loaded into a crossed dipole trap, where the atoms are further cooled by forced evaporation [40]. After cooling at magnetic field $B=24 \mathrm{G}$ for 5 seconds, we quickly switch the field near the Feshbach resonance at $47.8 \mathrm{G}$ [41], where the evaporation continues at a scattering length of $a=300 \quad a_{0}$ for one second and yields an almost pure condensate with $N=10^{4}$ atoms. At this point, the trap frequencies are $\left(\omega_{x}, \omega_{y}, \omega_{z}\right)=2 \pi \times(12,30,70) \mathrm{Hz}$ and the peak density is $2 \times 10^{13} \mathrm{~cm}^{-3}$. Use of this narrow Feshbach resonance, which has a width of $160 \mathrm{mG}$, increases the sensitivity of the scattering length to the vector light shift, allowing a given change in scattering length to be achieved with lower laser intensity and therefore longer lifetime for the quantum gas.

For cesium atoms one possible magic wavelength is $869.7 \mathrm{~nm}$ with a $\sigma^{+}$polarized laser, which is far-detuned from atomic transitions but maintains a large vector polarizability [Fig. 2(a)]. To confirm the magic wavelength experimentally, we measure the atomic polarizability at different wavelengths. To do so we displace the OFR beam from the center of the BEC and measure the change in the center of mass position of the condensate [30]. At the magic wavelength, the beam does not move the condensate. We find the magic wavelength to be $\lambda_{M}=869.73(2) \mathrm{nm}$ [Fig. 2(b)]. At the intensity $I=225 \mathrm{~W} / \mathrm{cm}^{2}$ used for most of this work, we estimate that the residual dipole potential $k_{B} \times 1 \mathrm{nK}$ is negligible compared to our typical chemical potential of $k_{B} \times 10 \mathrm{nK}$, where $k_{B}$ is the Boltzmann constant. With uniform exposure to this intensity, the loss induced by OFR is well explained by a one-body decay with a time constant of 0.63(2) s [Fig. 2(c)]. We compare
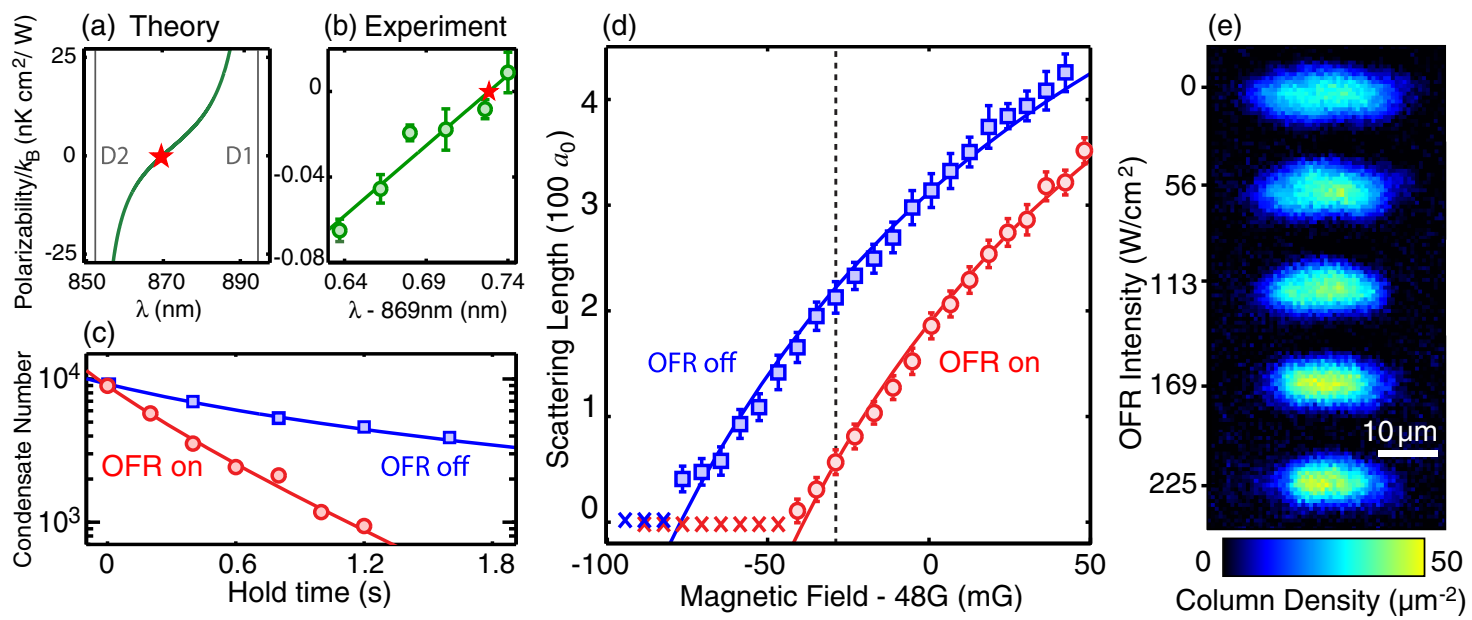

FIG. 2 (color online). Stable optical control of scattering length at a magic wavelength $\lambda_{M}$. (a) Theoretical polarizability of Cs atoms in the absolute ground state for $\sigma^{+}$polarization [30]. The star marks the magic wavelength where polarizability is zero. (b) Measured polarizability (circle). A linear fit yields $\lambda_{M}=869.73(2) \mathrm{nm}$ (star). (c) Number of condensed atoms remaining over time with (circle) and without (square) exposure to OFR at a magnetic field of $48.19 \mathrm{G}$. We fit the decay dynamics (solid curves) and find that OFR exposure adds a one-body loss process with a time constant of $0.63(2) \mathrm{s}$ [30] at intensity $I=225 \mathrm{~W} / \mathrm{cm}^{2}$. (d) Scattering length $a$ determined from the free expansion of BECs with (circle) and without (square) exposure to the OFR laser. When the scattering length becomes negative $(a<0)$ the condensate collapses (cross). The solid curves derive from a single fit to all $a>0$ data using Eq. (2), which yields $\Delta=157(3) \mathrm{mG}, B_{0}=47.766(4) \mathrm{G}$, and $\beta I=-38(1) \mathrm{mG}$. (e) In situ images of BECs at $47.97 \mathrm{G}$ [dashed line in panel (d)] after ramping on the intensity of the OFR laser over $200 \mathrm{~ms}$. Each image is the average of 10 trials. All error bars show standard error. 
this time constant to the best previous OFR result [26], which is limited by two-body loss. After scaling their result to the density of our gas and the same change in scattering length, we find that our scheme provides more than an order of magnitude improvement in the lifetime.

To precisely determine the change of scattering length, we allow the BEC to freely expand with and without exposure to the OFR laser and measure the size of the gas after expansion $[42,43]$. During free expansion the interaction energy, which is proportional to $a$, is converted to kinetic energy which we measure via the expanded cloud size [30]. Figure 2(d) shows the shift of scattering length induced by the laser near the $47.8 \mathrm{G}$ Feshbach resonance. We fit the scattering lengths with a theoretical model [14]

$$
a(I)=a_{\mathrm{bg}}\left[1-\frac{\Delta}{B(I)-B_{0}}\right]
$$

where $B(I)=B_{\text {ex }}+\beta I$ is the effective magnetic field including the contribution $\beta I$ from OFR [Eq. (1)] and the external field $B_{\mathrm{ex}}, a_{\mathrm{bg}} \approx 950 a_{0}$ is the background scattering length at this Feshbach resonance [44], $\Delta$ is the width of the resonance, and $B_{0}$ is the resonance position. The fit yields $\beta I=-38(1) \mathrm{mG}$, sufficient to shift from $a=180 a_{0}$ to zero.

The long lifetime of BECs exposed to the OFR laser allows us to corroborate the change of scattering length based on in situ measurements of the density profile. We slowly ramp on the OFR beam to four different final intensities over $200 \mathrm{~ms}$ and measure the resulting density profiles [Fig. 2(e)]. Higher intensities of the OFR laser shrink the BEC and increase its density, consistent with weakening the repulsive interactions. A mean-field analysis yields scattering lengths in excellent agreement with the free expansion measurement [30].
The stability of this scheme enables us to explore temporal and spatial control of interactions in a quantum gas. We first perform interaction modulation spectroscopy by recording the response of the BEC to an OFR beam with oscillating intensity. We perform these experiments either in the bulk, with the vertical trap frequency $\omega_{z}$ increased to $2 \pi \times 470 \mathrm{~Hz}$, or in a weak one-dimensional optical lattice in the horizontal plane with spacing $532 \mathrm{~nm}$ and lattice depth $h \times 9.28 \mathrm{kHz}$, in which the system remains a superfluid [45]. For this experiment only, we introduce the optical lattice in order to test the consequences of modulated interactions in a lattice gas, as discussed in many theoretical proposals $[1,2,7]$. Combining the experiments in both geometries, we observe a variety of resonance features over a wide range of time scales, as shown in Fig. 3, highlighting the versatility of this technique.

At lower frequencies we observe excitations in the trap and in the optical lattice. The oscillating scattering length drives parity-conserving transitions to the second excited state in the vertical trap at $890(10) \mathrm{Hz}$ and the second excited band of the optical lattice at $8.18(2) \mathrm{kHz}$. This demonstrates that the plentiful theoretical proposals [1-7] which require rapid oscillation of scattering length in the bulk or in the lattice are well within reach of our scheme.

At higher frequencies the oscillating intensity of the OFR beam induces formation of molecules. We identify a broad resonance at $133(7) \mathrm{kHz}$ corresponding to a virtual molecular state embedded in the continuum, as well as narrow resonances at 197(1) kHz and 11.649(2) MHz corresponding to a weakly bound Feshbach molecular state and a deeply bound van der Waals molecular state, respectively. All observed resonance positions are in excellent agreement with theoretical calculations [30]. These resonances provide direct evidence that OFR can access interaction physics on time scales as short as $10 \mathrm{~ns}$.

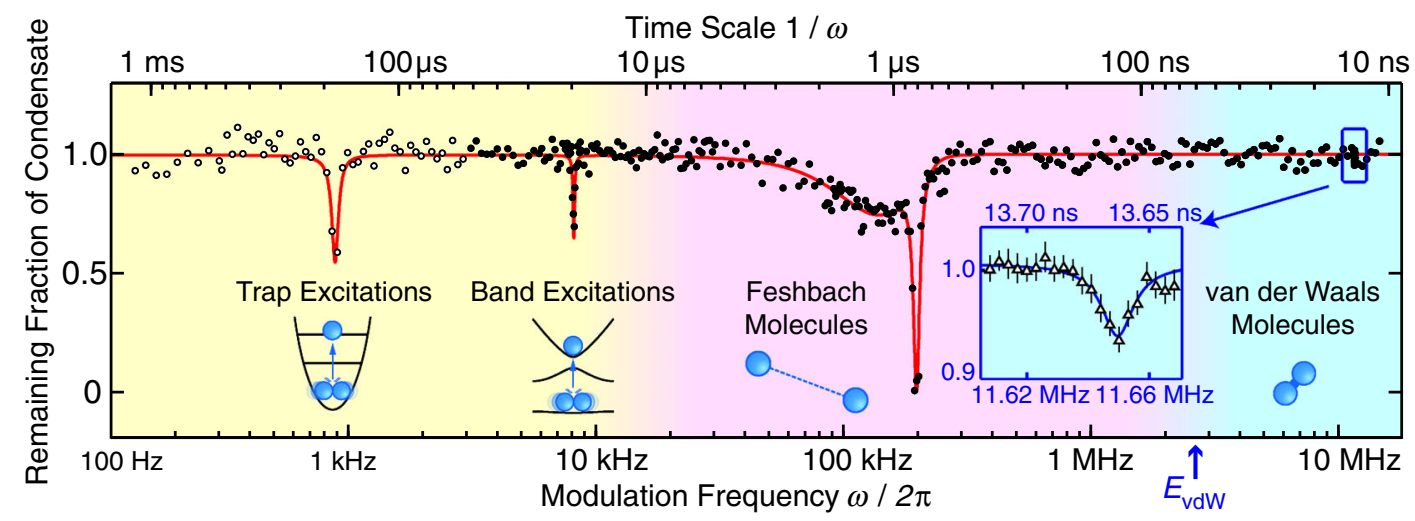

FIG. 3 (color online). Interaction modulation spectroscopy. The BEC at $47.976 \mathrm{G}$ is exposed for a hold time $t$ to the OFR laser, which is intensity modulated at frequency $\omega / 2 \pi$. We measure the number of condensed atoms remaining after exposure, normalized to the off-resonant number, under three conditions: (open circle) $t=100 \mathrm{~ms}$ with no optical lattice, (filled circle) $t=20 \mathrm{~ms}$ with a one dimensional optical lattice of depth $h \times 9.3 \mathrm{kHz}$, (open triangle) $t=500 \mathrm{~ms}$ with no optical lattice. Resonances are observed at $0.89(1)$, 8.18(2), 133(7), 197(1), and 11 649(2) kHz, determined from fits (solid curve) to each resonance (Gaussian for 133 kHz, Lorentzian for others). The illustrations indicate the nature of each resonance. The van der Waals energy scale is $E_{\mathrm{vdW}}=h \times 2.7 \mathrm{MHz}$ for Cs molecules. 


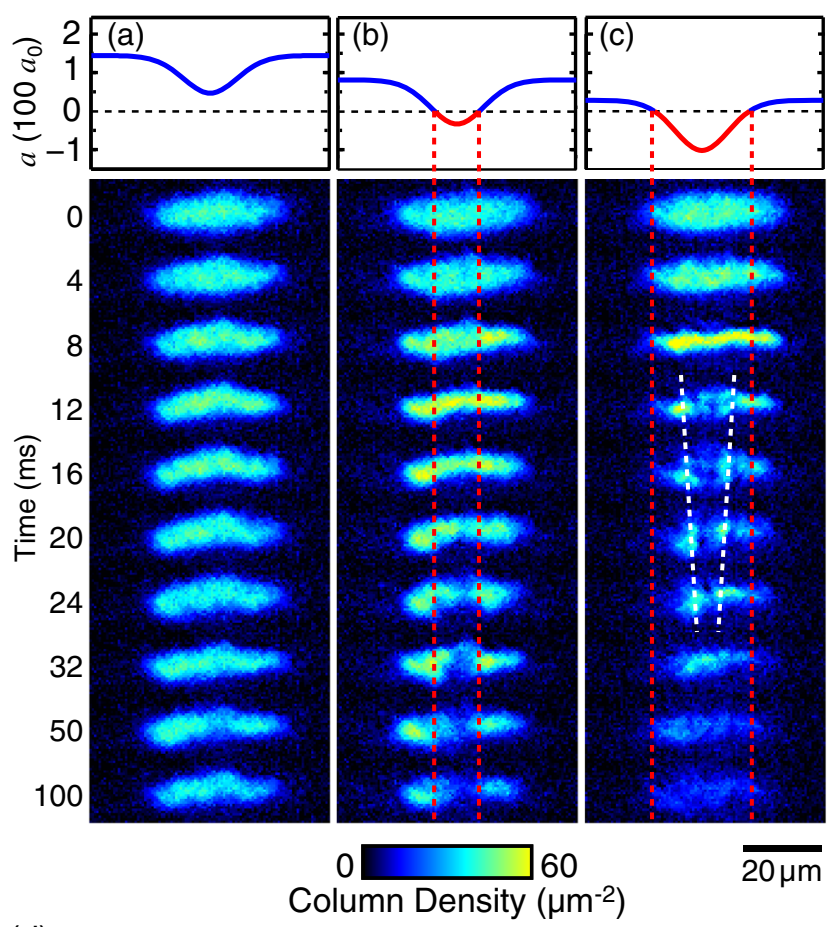

(d)

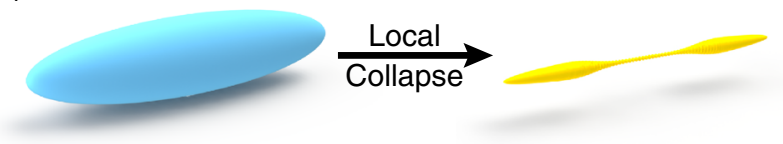

FIG. 4 (color online). Condensate dynamics with spatially modulated interactions. Time series of in situ images of BECs with $N=12000$ atoms after a quench from uniform $a=200 a_{0}$ at $47.965 \mathrm{G}$ to the spatially modulated $a$ shown in the top panels. The OFR beam has peak intensity of $115 \mathrm{~W} / \mathrm{cm}^{2}$ and a waist of $14 \mu \mathrm{m}$, while the final magnetic fields are (a) $47.949 \mathrm{G}$, (b) $47.935 \mathrm{G}$, and (c) $47.925 \mathrm{G}$. Each image is the average of 6 or 7 trials. The red dashed lines show where $a$ equals zero. The white dashed lines in panel (c) guide the eye to the motion of the solitonic wave towards the trap center. (d) Illustration of the local collapse dynamics, in which the initial BEC (left) undergoes transverse compression followed by localized central collapse (right).

Next, we demonstrate spatial modulation of the interaction strength within a quantum gas. For this experiment we employ an OFR beam which is small compared to the size of the $\mathrm{BEC}$, leading to a reduced scattering length only in the center of the gas. After preparing the BEC we quickly turn on the OFR beam and simultaneously change the magnetic field. We study the subsequent dynamics of the sample by measuring its in situ density profile over time (Fig. 4). For example, when the interactions remain repulsive throughout the condensate, we observe collective excitations for the duration of the experiment [Fig. 4(a)]. For all images the small distortion at the center of the gas results from the dipole potential due to slightly nonuniform laser polarization [30].

Intriguing quantum dynamics occur when the interactions become locally attractive. When the scattering length is negative in a small region near the center of the trap [Fig. 4(b)], we observe a brief period of transverse compression followed by a rapid drop in central density between 20 and $32 \mathrm{~ms}$ after the quench, signaling local collapse of the condensate [illustrated in Fig. 4(d)]. A large fraction of the sample survives at the edges for more than $100 \mathrm{~ms}$. With even stronger attractive interactions [Fig. 4(c)], faster central collapse occurs after $8 \mathrm{~ms}$. Subsequently, the dense remnants at the edge of the sample move toward the center of the trap [see white dashed lines in Fig. 4(c)]. Beyond $32 \mathrm{~ms}$ only thermal gas survives, indicating that the remnants have undergone further collapse. This behavior is reminiscent of bright matter wave solitons [46-48]; the remnants form at a small negative scattering length but become unstable as they move toward the center where the scattering length is more negative. Further investigation into the nature of these remnants provides an intriguing course for future work. Moreover, the variety of behaviors observed in this experiment establishes the richness of the quantum dynamics accessible with space dependent interactions.

In conclusion, we implement a generic scheme for optically controlling interactions in stable quantum gases. This scheme overcomes the key challenges encountered by past approaches to OFR. Fast and local control of interactions in a quantum gas enables studies of quantum dynamics in new regimes and has great potential in the fields of quantum simulation and engineering, opening a new frontier of interaction-driven quantum phenomena.

We thank Y. T. Chen for assistance in the early stages of the experiment, P.S. Julienne for coupled-channel calculations of Cs molecular structure, and C. V. Parker for helpful discussions. L. W. C. is supported by the NDSEG Fellowship. L.-C.H. is supported by the Grainger Fellowship and the Taiwan Government Scholarship. This work was supported by NSF MRSEC (DMR1420709), NSF Grant No. PHY-1511696 and AROMURI Grant No. W911NF-14-1-0003.

*Corresponding author. lwclark@uchicago.edu

[1] J. Gong, L. Morales-Molina, and P. Hänggi, Phys. Rev. Lett. 103, 133002 (2009).

[2] S. Greschner, G. Sun, D. Poletti, and L. Santos, Phys. Rev. Lett. 113, 215303 (2014).

[3] F. K. Abdullaev, J. G. Caputo, R. A. Kraenkel, and B. A. Malomed, Phys. Rev. A 67, 013605 (2003).

[4] H. Saito and M. Ueda, Phys. Rev. A 70, 053610 (2004).

[5] K. Staliunas, S. Longhi, and G. J. de Valcárcel, Phys. Rev. Lett. 89, 210406 (2002).

[6] E. R. F. Ramos, E. A. L. Henn, J. A. Seman, M. A. Caracanhas, K. M. F. Magalhães, K. Helmerson, V. I. Yukalov, and V.S. Bagnato, Phys. Rev. A 78, 063412 (2008). 
[7] F. K. Abdullaev, P. G. Kevrekidis, and M. Salerno, Phys. Rev. Lett. 105, 113901 (2010).

[8] M. I. Rodas-Verde, H. Michinel, and V. M. Pérez-García, Phys. Rev. Lett. 95, 153903 (2005).

[9] J. Belmonte-Beitia, V. M. Pérez-García, V. Vekslerchik, and P. J. Torres, Phys. Rev. Lett. 98, 064102 (2007).

[10] M. J. Hartmann and M. B. Plenio, Phys. Rev. Lett. 100, 070602 (2008).

[11] M. Salerno, V. V. Konotop, and Y. V. Bludov, Phys. Rev. Lett. 101, 030405 (2008).

[12] R. Balbinot, A. Fabbri, S. Fagnocchi, A. Recati, and I. Carusotto, Phys. Rev. A 78, 021603 (2008).

[13] S. Inouye, M. R. Andrews, J. Stenger, H.-J. Miesner, D. M. Stamper-Kurn, and W. Ketterle, Nature (London) 392, 151 (1998).

[14] C. Chin, R. Grimm, P. Julienne, and E. Tiesinga, Rev. Mod. Phys. 82, 1225 (2010).

[15] P. O. Fedichev, Y. Kagan, G. V. Shlyapnikov, and J. T. M. Walraven, Phys. Rev. Lett. 77, 2913 (1996).

[16] J. L. Bohn and P. S. Julienne, Phys. Rev. A 56, 1486 (1997).

[17] F. K. Fatemi, K. M. Jones, and P. D. Lett, Phys. Rev. Lett. 85, 4462 (2000).

[18] M. Theis, G. Thalhammer, K. Winkler, M. Hellwig, G. Ruff, R. Grimm, and J. H. Denschlag, Phys. Rev. Lett. 93, 123001 (2004).

[19] T. Zelevinsky, M. M. Boyd, A. D. Ludlow, T. Ido, J. Ye, R. Ciuryło, P. Naidon, and P. S. Julienne, Phys. Rev. Lett. 96, 203201 (2006).

[20] K. Enomoto, K. Kasa, M. Kitagawa, and Y. Takahashi, Phys. Rev. Lett. 101, 203201 (2008).

[21] R. Yamazaki, S. Taie, S. Sugawa, and Y. Takahashi, Phys. Rev. Lett. 105, 050405 (2010).

[22] S. Blatt, T. L. Nicholson, B. J. Bloom, J. R. Williams, J. W. Thomsen, P. S. Julienne, and J. Ye, Phys. Rev. Lett. 107, 073202 (2011).

[23] H. Wu and J. E. Thomas, Phys. Rev. Lett. 108, 010401 (2012).

[24] M. Yan, B. J. DeSalvo, B. Ramachandhran, H. Pu, and T. C. Killian, Phys. Rev. Lett. 110, 123201 (2013).

[25] D. M. Bauer, M. Lettner, C. Vo, G. Rempe, and S. Dürr, Nat. Phys. 5, 339 (2009).

[26] D. M. Bauer, M. Lettner, C. Vo, G. Rempe, and S. Dürr, Phys. Rev. A 79, 062713 (2009).

[27] Z. Fu, P. Wang, L. Huang, Z. Meng, H. Hu, and J. Zhang, Phys. Rev. A 88, 041601 (2013).

[28] R. A. Williams, M. C. Beeler, L. J. LeBlanc, K. JiménezGarcía, and I. B. Spielman, Phys. Rev. Lett. 111, 095301 (2013).
[29] F. Le Kien, P. Schneeweiss, and A. Rauschenbeutel, Eur. Phys. J. D 67, 92 (2013).

[30] See Supplemental Material at http://link.aps.org/ supplemental/10.1103/PhysRevLett.115.155301 for more details on the experimental methods and data analysis, as well as theoretical analysis of the expected performance of OFR, which includes Refs. [31-37].

[31] A. Widera, O. Mandel, M. Greiner, S. Kreim, T. W. Hänsch, and I. Bloch, Phys. Rev. Lett. 92, 160406 (2004).

[32] M. Lu, N. Q. Burdick, S. H. Youn, and B. L. Lev, Phys. Rev. Lett. 107, 190401 (2011).

[33] K. Aikawa, A. Frisch, M. Mark, S. Baier, A. Rietzler, R. Grimm, and F. Ferlaino, Phys. Rev. Lett. 108, 210401 (2012).

[34] A. Frisch, M. Mark, K. Aikawa, F. Ferlaino, J. L. Bohn, C. Makrides, A. Petrov, and S. Kotochigova, Nature (London) 507, 475 (2014).

[35] K. Baumann, N. Q. Burdick, M. Lu, and B. L. Lev, Phys. Rev. A 89, 020701 (2014).

[36] C. J. Pethick and H. Smith, Bose-Einstein Condensation in Dilute Gases (Cambridge University Press, Cambridge, England, 2002).

[37] S. T. Thompson, E. Hodby, and C. E. Wieman, Phys. Rev. Lett. 95, 190404 (2005).

[38] M. Cetina, M. Jag, R. S. Lous, J. T. M. Walraven, R. Grimm, R. S. Christensen, and G. M. Bruun, arXiv:1505.00738 [Phys. Rev. Lett. (to be published)].

[39] C. Weitenberg, M. Endres, J. F. Sherson, M. Cheneau, P. Schauß, T. Fukuhara, I. Bloch, and S. Kuhr, Nature (London) 471, 319 (2011).

[40] C.-L. Hung, X. Zhang, N. Gemelke, and C. Chin, Phys. Rev. A 78, 011604 (2008).

[41] A. D. Lange, K. Pilch, A. Prantner, F. Ferlaino, B. Engeser, H.-C. Nägerl, R. Grimm, and C. Chin, Phys. Rev. A 79, 013622 (2009).

[42] Y. Castin and R. Dum, Phys. Rev. Lett. 77, 5315 (1996).

[43] T. Volz, S. Dürr, S. Ernst, A. Marte, and G. Rempe, Phys. Rev. A 68, 010702 (2003).

[44] M. Berninger, A. Zenesini, B. Huang, W. Harm, H.-C. Nägerl, F. Ferlaino, R. Grimm, P. S. Julienne, and J. M. Hutson, Phys. Rev. A 87, 032517 (2013).

[45] L.-C. Ha, L. W. Clark, C. V. Parker, B. M. Anderson, and C. Chin, Phys. Rev. Lett. 114, 055301 (2015).

[46] K. E. Strecker, G. B. Partridge, A. G. Truscott, and R. G. Hulet, Nature (London) 417, 150 (2002).

[47] L. Khaykovich, F. Schreck, G. Ferrari, T. Bourdel, J. Cubizolles, L. D. Carr, Y. Castin, and C. Salomon, Science 296, 1290 (2002).

[48] S. L. Cornish, S. T. Thompson, and C. E. Wieman, Phys. Rev. Lett. 96, 170401 (2006). 